\title{
Carers and the NHS
}

There has been much discussion on the kind of workforce needed to deliver the NHS of the 21 st century. It should, therefore, be surprising that the UK's largest health and care workforce has so far been overlooked by these discussions. However, if you look at the job descriptions for these workers, it is easy to see that, for them, being forgotten is not unusual. Working hours: 24 hours a day, 7 days a week. Training: none. Support: none. Career prospects: none. Holidays: none. Pay: up to $£ 1.50$ per hour (for those lucky enough to qualify for benefits). With pay and conditions this poor, no wonder family carers are estimated to save the NHS $£ 67$ billion $^{1}$ or more per annum.

The scale of this contribution is staggering. Each year, 2 million of us move in and out of unpaid caring for a sick, disabled or older relative or friend who is unable to manage on their own. There are around 6 million carers in the UK at any one time and 1.2 million of those are caring for over 50 hours each week. ${ }^{2}$ The perception that the majority of carers are women is belied by the $42 \%$ of male carers uncovered by the last census. In fact, the majority of us will either give or receive care at some point in our lives.

Caring can take its toll both physically and emotionally, with the carer's own health and wellbeing suffering as a consequence of their caring responsibilities. Half of carers report a longstanding illness, with $72 \%$ of people caring for 50 or more hours reporting that caring adversely affects their health, ${ }^{3}$ and many live on low incomes and in poor housing. In 2002, Torbay Council and Manchester PSSRU used General Health Questionnaires (GHQ) with 68 randomly selected carers. Despite over half of carers saying that they were in good health, GHQ scores at referral/commencement suggested that nearly all suffered significant emotional distress, which was reduced by $21 \%$ through low-cost Carer Support Worker interventions hosted by GP practices. ${ }^{4}$

Ten per cent of the average GP practice's population are carers, and so the support of primary healthcare professionals can make a dramatic difference to the health and wellbeing of carers. In this winter's snowy weather an older couple died and lay undiscovered for up to 5 days. The husband, sole carer for his wife who used a wheelchair, was believed to have died first, followed shortly afterwards by his wife, who no longer had anyone to support her. This tragic incident was the extreme and hopefully exceptional consequence of the alarmingly high incidence of isolation and lack of emergency support that affects thousands of carers. GP practices play a vital role in identifying carers, recognising their health needs and putting them in touch with the UK's network of more than 150 Carers' Centres.

Carers, even those who are very elderly, are often left to carry out lifting and handling that even paid professionals would not be allowed to consider without the aid of a hoist. In some areas, NHSbased work is making a difference to the health of this substantial group of vulnerable people. Carers Gloucester's Surgery Link project provides trained team members to visit surgeries once every 4-6 weeks, in order to liaise with staff via a nominated Carers Lead; provide information; take referrals to the carers' centre; and attend outreach sessions. The Carers' Resource in Harrogate, Craven and Airedale provides carers services in partnership with memory clinics and palliative care professionals to ensure that the carers of people with dementia and life limiting illnesses are supported alongside the patient. Carer Support Officers are also linked with Single Assessment Process (SAP) teams to ensure carers' needs are addressed through the SAP system. Some GP surgeries make home and telephone support more easily available to carers who cannot leave the person they are caring for alone while others have appointments reserved for carers.

In addition to mitigating the health risks experienced by carers themselves, support for carers has knock-on benefits for the person who needs care. Most carers want to continue caring and will do so as long as they remain able. Support to care has been shown to delay the use of expensive hospital or residential care by 500 days over a 10-year period. ${ }^{5}$ Some PCTs have saved pennies by withdrawing the provision of basic equipment to carers, such as disposable gloves and aprons for carers dealing with multiple daily instances of incontinence, but such efficiencies are likely to be costly as carers fall ill or become ever more ground down by the indignities of caring unsupported. PCTs such as Nottingham PCT, Knowsley PCT, Cornwall and Isles of Scilly PCT, and Coventry PCT have been investing over $£ 1$ million per annum in carer support for several years ${ }^{6}$ but recognition of the strong business and health cases for carer support are yet to become the norm.

Carers often report that support succeeds or fails at the interfaces between the NHS and social care services. Those areas performing strongly on carers' support are often those who are strong on joint commissioning. There is anecdotal evidence and an ongoing research programme aimed at establishing the costbenefits of supporting carers at the point of hospital discharge. Where discharge teams work with staff from Carers' Centres, results suggest that discharge can be accelerated and is less likely to involve readmissions.

There are even 175000 children, aged between 5 and 18 years, in substantial caring roles. Often, they care for parents who have disabilities, mental or physical illnesses, or substance misuse problems. Like adult carers, some young carers carry out manual handling tasks that damage their backs. The Princess Royal Trust for Carers has uncovered worryingly high levels of self-harm and other emotional health problems among the young carers who use the Trust's online support service (www.youngcarers.net). Other young carers report back problems due to lifting their parent or heavy equipment such as wheelchairs. Some accompany parents with communication problems, or with little or no knowledge of English, to surgery 
appointments, and are called upon to interpret. This can expose the young carer to stressful or age-inappropriate information, which is to the detriment of both parent and child.

The National Carers' Strategy, ${ }^{7}$ launched in 2008, began with the largest ever consultation of carers and found that carers placed recognition and the provision of information as some of their highest priorities for change. The first ambition of the Strategy is that, by 2018 , carers will routinely expect to be 'respected as expert care partners' by NHS and other professionals. The Strategy includes $£ 150$ million of new NHS money for primary care trusts, although research suggests only about $20 \%{ }^{6}$ of that was actually spent on carers.

The government's laudable aspirations for carers will, of course, only be made reality through the engagement of local services. With this in mind, the RCGP and The Princess Royal Trust for Carers have launched a new resource to support carers in primary care settings. Supporting Carers: An Action Guide for GPs and Their Teams is available at www.carers.org/professionals. ${ }^{4}$ The guide has been piloted in 20 GP practices and evaluated by Sheffield University. It aims to help primary care staff provide earlier and more effective interventions for the hundreds of unrecognised carers that the average practice comes into contact with each year. There are numerous examples of inventive solutions and it is intended that the guide will be constantly developed as new evidence of good practice emerges.

One issue not addressed by the Carers' Strategy, but which remains central for carers and professionals alike, is the apparent conflict between NHS confidentiality requirements and the need for carers to be informed about their relative's health condition and treatment, in order to care more safely and effectively. Many carers manage and administer medication, feeding tubes, colostomy bags, or other medical equipment for which they need support and training.
Carers of people with mental health or substance misuse problems may perform few physical tasks, but provide a great deal of emotional support, including helping to ensure that someone stays safe, and responding to incidents of self-harm or overdose. There have been incidences of older parents left unsupported in unsafe situations, and not listened to by professionals as their relative's health deteriorates.

As NHS resources shrink, we will rely even more on the caring contributions of unpaid family carers. Unmanageable, 24-7 caring roles carried out in isolation are not sustainable. Instead, the NHS will need to help more people to sustain smaller caring roles, which they can manage alongside paid employment and while maintaining their own health. Primary care will be at the front line of achieving this, but success will pay huge dividends to carers, those they care for and the local health economy.

\section{Alex Fox, Nigel Sparrow and Jo Webber}

\section{REFERENCES}

1. Moullin S. Just care: a fresh approach to adult services. London, Institute for Public Policy Research, 2008.

2. UK Census 2001. London, Office for National Statistics, 2002.

3. Maher J, Green H. Carers 2000. London: Office for National statistics, 2002. http://www.statistics.gov.uk/downloads/theme_health/c arers2000.pdf (accessed 16 May 2010).

4. The Princess Royal Trust for Carers and RCGP. Supporting carers: an action guide for general practitioners and their teams. London, 2008. http://www.carers.org/professionals (accessed 16 May 2010).

5. Mittelman MS, Ferris SH, Shulman E, et al. Family intervention to delay nursing home placement of patients with Alzheimer's disease. JAMA 1996; 276(21):1725-31.

6. The Princess Royal Trust for Carers. Tough breaks for carers. A report into Primary Care Trusts and delivery of the Carers' Strategy. London, 2010.

http://www.carers.org/professionals/health/articles/nhsgiven-150m-to-support-carers,5615,PR.html (accessed 16 May 2010).

7. Department of Health. Carers at the heart of 21st century families and communities. A caring system on your side. A life of your own. London: Department of Health, 2008. http://www.dh.gov.uk/en/Publicationsandstatistics/Publi cations/PublicationsPolicyAndGuidance/DH_085345 (accessed 16 May 2010).

DOI: 10.3399/bjgp10X509757 